\title{
Social Media Analytics using Qlik Connectors
}

\author{
Siddhartha Ghosh, Kandula Neha
}

\begin{abstract}
In the digital age high-end computers are used for storing an increased amount of data, which might not be possible a decade ago. These computers run on sophisticated software algorithms producing fast insights needed to make fact-based decisions. By implementing the science of numbers, data and analytical discovery to work together, it has been found that what we think or believe; produce answers to questions we never thought to ask. That's the power of analytics. Information has been scrutinize as a powerful weapon, and analytics is the forge that creates it. Analytics changes everything, not just in the world of business, but also in science, sports, health care and in few field where enormous data are collected. The combination of analytics and connectors into social media gives an immense accuracy and popularity throughout different forms of business. This paper is the preliminary analysis of few areas considered from social media like Face book, Twitter etc, where data is gathered and analytics is drawn with the help of connectors through Qlik.
\end{abstract}

Key Words : Analytics, Connectors, Face book, Traditional Methods, Visualization

\section{INTRODUCTION}

Analytics : A procedure which is followed in data for discovering and communicating the meaningful patterns. It is concerned which turns raw data into insight for better decisions. Where the Application relies on statistics and often involve in studying past historic data to research potential trends. Specially useful in the areas which record a huge amount of data or information.

It provides meaningful information which are hidden within large quantities of data which also helps us to find hidden patterns, from consumer behaviours, athlete and team performance, to finding connections between activities and diseases. This can change how one looks at the world, and usually for the better.

What happened? How or why did it happen? What's happening now? What is likely to happen next?

Google Analytics : An internet Site where traffic analysis application that provides real-time statistics and analysis of user interaction with the website. It enables owners to analyze their visitors, with the objective of interpreting and optimizing website's portrayal and monitors digital media and referring upstream web destinations, banner and contextual advertisements, e-mail and integrates with other products.

\section{WHY ANALYTICS IS IMPORTANT ?}

Analysis is focused on understanding the past - what, why and what will happen next. Analization of data can uncover correlations and patterns. There's less need to rely on guesses or intuition. It can help answer questions.

With faster and more powerful computers, opportunity overflows for the use of big data analytics. Whether it's determining credit risk, developing new medicines, finding more efficient ways to deliver products and services, preventing fraud, un covered threats in cyber or retaining the most valuable customers, analytics can help understand an organization - and the world around it.

Applications of Analytics : Most of the Analytics Applications used to measure and improve the performance of business applications which generate reports of the business growth for more profits in future.

1. Marketing Optimization : Marketing organizations use analytics to determine the outcomes of campaigns and to guide decisions for investment for their growth. Marketers use Google Analytics as showcases an example of free popular tool of analytics. for improved performance these kinds of interactions are needed.

2. People Analytics :People Analytics which is also known as Workforce HR analytics it is used to understand how people work and how companies are been managed.

3. Risk Analytics : These analyses are carry out extensively used in financial institutions like Online payment Gateway companies to analyse if a transaction was genuine or fraud.

4. Digital Analytics : Digital analytics for business and technical activities which define, create, collect, verify or transform digital data into reporting, research, analyses, recommendations, optimizations, predictions, and automations. This also includes the SEO (search engine optimization) where the keyword search is tracked and that data is used for marketing purposes.

5. Security Analytics : Security analytics refers to information technology (IT) to gather and analyze security events to understand and analyze events that pose the greatest risk.

6. Software Analytics : Software analytics is the process of collecting information about the way a piece of software is used and produced.

These traditional analytics tools, data is most often broken down into graphs, charts, lists, trends, and other tabular formats that attempt to provide actionable insight.
Revised Version Manuscript Received on 16 September, 2019.

Dr. Siddhartha Ghosh, Dept of Computer Science \& Engineering, Vidya Jyothi Institute of Technology, Hyderabad, India.

Kandula Neha, Dept of Computer Science \& Engineering, Vidya Jyothi Institute of Technology, Hyderabad, India.

Published By: 


\section{III QLIK CONNECTORS - A NEW TOOL FOR ANALYTICS}

QLIK : QlikView has just launched a new tool called Qlik Sense provide self-service data visualization and business intelligence capabilities for individuals and organizations. Qlik Sense offers a simplified user interface, drag-and-drop visualizations, and the ability to load data without manually editing scripts. QlikView is the Business Intelligence platform for turning data into knowledge. It enables users to gain unexpected business insights where which data is associated and what data is not related. They can conduct direct and indirect searches across all data anywhere in the application globally or within a single field.

\section{What is Qlik Sense?}

Qlik Sense is a platform for data analysis. With Qlik Sense you can analyze data and make data discoveries on your own. You can share knowledge and analyze data in groups and across organizations. Qlik Sense lets you ask and answer your own questions and follow unique way to insight and enables you and your colleagues to reach decisions collaboratively.

\section{Why use Qlik with Google Analytics data?}

Qlik Easily integrate Google Analytics information into your analysis:

- Flexibility to combine data sources - Qlik's associative model seamlessly combines data from Google Analytics, social media channels and few data sources in to data applications.

- Rapid deployment - The Qlik Google Analytics Connector can be configured and placed into production quickly, new insights will be gained within no time - not weeks or months.

- View all of your websites in one place - It can be difficult to see a complete picture in a single view, especially when we ponder websites side-by-side. Qlik gives liberates you to handpick the KPIs that are important and so you can view everything in one place. No more wasting time switching between charts and website profiles.

Qlik Web Connectors is a comprehensive suite of pre-configured connectors for major social media and web-based data sources. It enables better decision making by allowing users to easily combine internal or on-premise information with cloud-based data sources. Qlik Web Connectors provide out-of-the-box connectors that are designed for specific application and data sources. This eradicate the need for any in-house connector development or maintenance and allows for quick implementation. Users can easily access popular Web-based services such as Facebook, Twitter, as well as online applications like Microsoft Dynamics CRM. These analytics through Qlik used for mapping and geo-analytics are near infinite and will vary widely depends on their business usage, there are a handful of features that can help any business will come right out of the gate. Including:

○ Visualization: Clustering and heat maps, KML layers, thematic maps, and routes.

○ Analytics: Complex filtering, numeric aggregates, dataset management, and charting.
○ Management: Territory management, automated assignment plans, mass updates, and custom markers and shapes.

Examples of these Connectors are :

Twitter : Qlik Twitter Connector can be used by a variety of customers gives you powerful insight into Twitter. The Qlik Twitter Connector allows you to load data through the Twitter API quickly and easily using specific usernames or search terms.

Facebook : Qlik offers two Facebook connectors that allow you to combine Face book data sets together.

- The Qlik Facebook Insights Connector provides metrics around the content of multiple Face book pages that you own or administer, allows you to analyze trends \& understands , demographics or consumption of content.

- The Qlik Facebook Fan Page Connector gives you insight into how people are engaged with a public Face book fan pages or groups, along with what they're posting and liking on the pages that you're analyzing.

The Qlik Google Analytics Connector provides users with a consistent, automated way to process this data so that timely strategic decisions can be made.

\section{QLIK GOOGLE ANALYTICS CONNECTOR - DATA VISUALIZATION EXPERIMENTAL RESULTS:}

This Qlik Sense application illustrates how Google Analytics data will be the best for customer's who use our site. Using the Qlik Connector, your Google Analytics data can easily be loaded into Qlik Sense providing a wealth of information. Analyzing the number of visits, page views, visit durations, bounces, locations, browsers devices and much more.

Qlik Facebook Connector : Brands comparison of Pepsi and Coco-cola FB Pages using the Qlik Facebook Group and Fan Pages Connector.

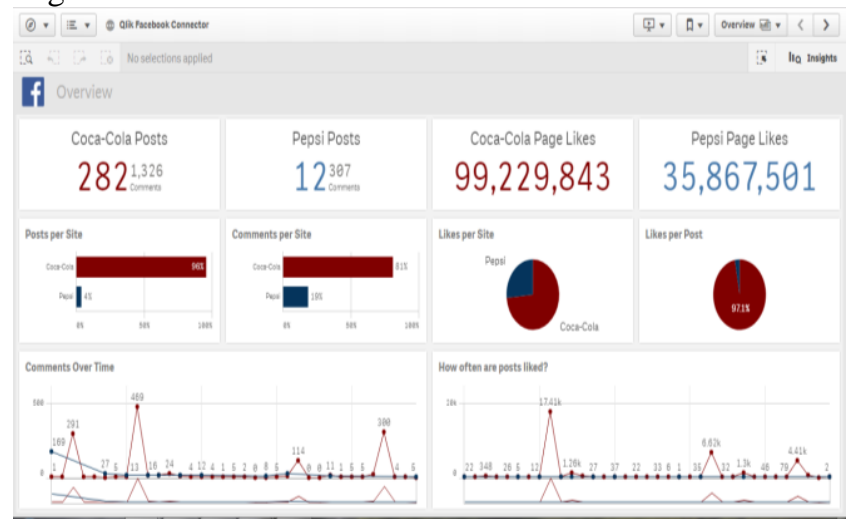

Fig 1: Over View of the method

Fig 1 Gives the Over View of both the Coco-Cola and Pepsi posts posted in their official Facebook page and their comments and post on per each post posted in their Site, where few charts like Bar Chart, Pie Chart, Time Graph and KPI Sheets are been used to give the insights of those pages in a detailed and brief manner.

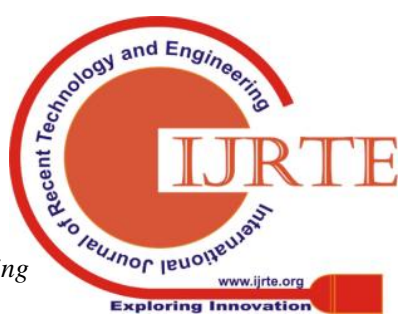




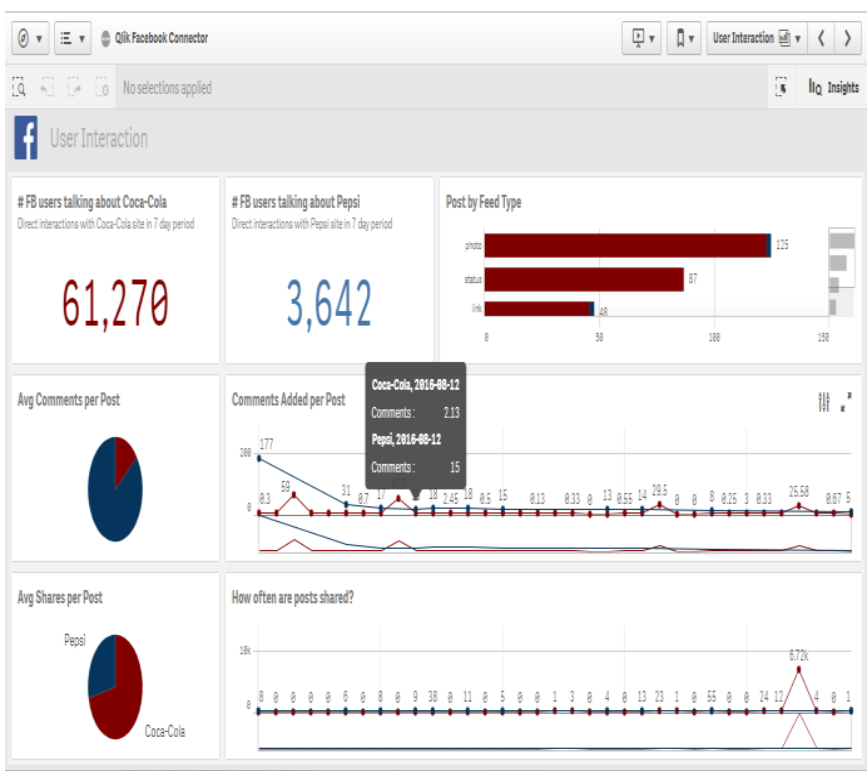

Fig 2 : User Interaction

Fig 2 describes that how the users are interacting among pages, sharing the digital content and commenting under the pictures or posts posted among those pages in over a period of time. It gives the over vie of the posts posted by both Coco-cola and Pepsi in their official FB pages which can be viewed by using the Hash tags which are in trend and use them while posting the content in the social media. These line graphs differentiates and compares both the products which are been trending in Social media from a period of time.

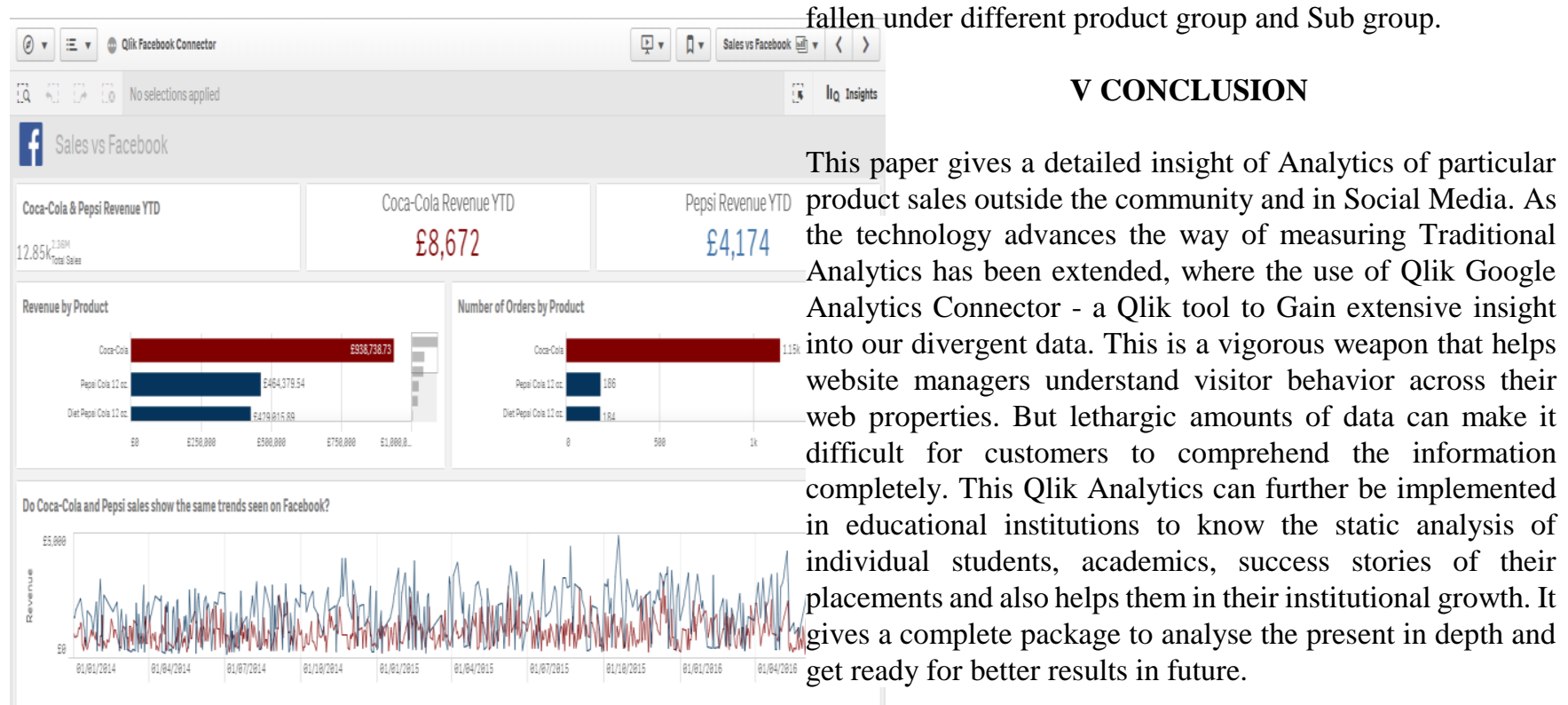

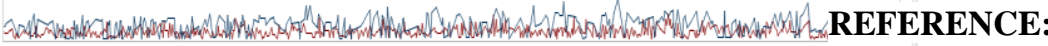

Fig 3 : Sales Vs Facebook

Fig 3 Gives the overview of details of Sales of those two branded products originally sold outside the hype created among Facebook by comparing their graphs logics among the social media using the few Graphs, Bar charts, and KPI Maps. The usage of above charts for both Coco-cola and Pepsi brands in social media gives the over all revenue spent on those products where insights are given to the companies about their future enhancements in sales and also sets new targets to achieve new goals and brings profits to the company and the brand.

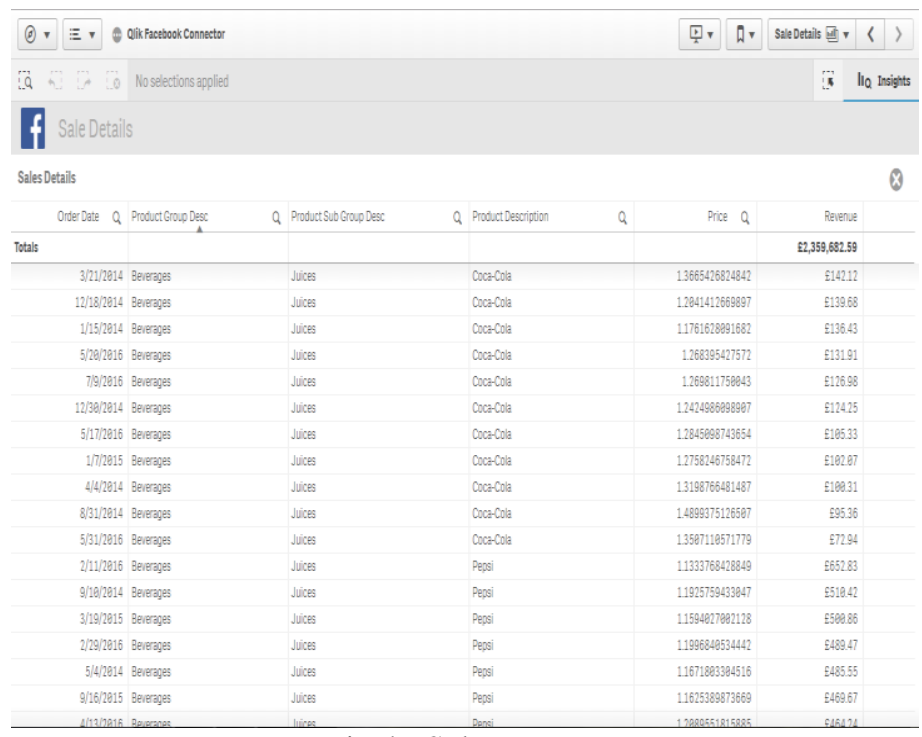

Fig 4 : Sales

Fig 4 gives the overall sales details and revenue of both Coco Cola and Pepsi in a period of time among different product groups and sub groups. This differentiation of Group and Subgroup gives an easy view of those products which are been used to give the insights. As we have considered both as products of similar kind which falls under same group as Beverages and Sub Group as Juices. If there are N number of products varieties taken for comparison then those will be fallen under different product group and Sub group.

This paper gives a detailed insight of Analytics of particular into our divergent data. This is a vigorous weapon that helps website managers understand visitor behavior across their web properties. But lethargic amounts of data can make it difficult for customers to comprehend the information completely. This Qlik Analytics can further be implemented placements and also helps them in their institutional growth. It gives a complete package to analyse the present in depth and

1. Dr Siddhartha Ghosh, Kandula Neha, Ballari Saha "Data Analytics in Academics, International Conference On Data Science And Analytics" (ICDSA'17). 23 September 2017.

2. Dr Siddhartha Ghosh, Kandula Neha, Ballari Saha "Enhancing Traditional Academic Processes Using Analytics", IICMR International Research Journal I4,Vol.12, Issue 1,Feb 2018,ISSN No.0975 2757 SJIF 2016:4.8. 
3. Dr Siddhartha Ghosh, Kandula Neha, Ballari Saha "Student Performance and Academic Pattern Analysis", International journal of Scientific Research in Science Engineering \& Technology (C) 2018 IJSRSET | Volume 4 | Issue 4 | Print ISSN: 2395-1990 | Online ISSN : 2394-4099, March - April 2018

4. Dr Siddhartha Ghosh, Kandula Neha, Ballari Saha "Measuring Health Status at workplace", Global Journal of Engineering Science and Researches GJESR ISSN 2348 - 8034 DOI- 10.5281/zenodo.1441091 Impact Factor- 5.070 September-2018.

5. Dr Siddhartha Ghosh, Kandula Neha, Ballari Saha "Aviation Safety Analysis using Data Analytics", JASC Journal of Applied Science and Computations, Volume VI, Issue 11, ISSN NO : 1076 - 5131, February 2019.

6. Budak C, Agrawal D, El Abbadi A (2011) Structural trend analysis for online social networks. Technical report UCSB/CS-2011-04, UCSB.

7. Larson K, Watson RT (2011) The value of social media: toward measuring social media strategies. In: Proc of 32nd international conference on information systems, Shanghai, China.

8. Zeng D, Chen H, Lusch R, Li S (2010) Social media analytics and intelligence. IEEE Intelligent Systems 25(6):13-16

\section{AUTHORS PROFILE}

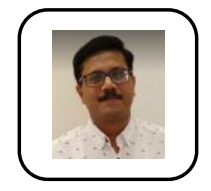

Dr. Siddhrtha Ghosh - Presently working as Professor in CSE Dept of Vidya Jyothi Institute of Technology. Has 18 years of teaching, 10 years of research and 2 years of industry experience. Has about 45 publications. Has own IBM USA Best innovative faculty award for Smart Cities in 2010. In 2018 he has been identified as Luminary of Qlik, Data Analytics company.

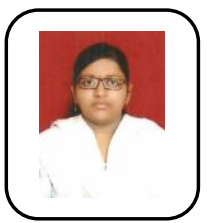

Kandula Neha - Presently working as Assistant Professor in CSE Dept of Vidya Jyothi Institute of technology. Has 2 years of experience in Research. Has about 11 publications. Recently certified in Qlik sense Business Analyst through QLIK. 